PUPT-1833

Imperial/TP/98-99/37

hep-th/9901101

\title{
A Non-supersymmetric Large N CFT from Type 0 String Theory
}

\author{
Igor R. Klebanov \\ Joseph Henry Laboratories, Princeton University, Princeton, New Jersey 08544, USA \\ Arkady A. Tseytlin ${ }^{\dagger}$ \\ Blackett Laboratory, Imperial College, London SW7 2BZ, U.K.
}

\begin{abstract}
We show that type $0 \mathrm{~B}$ theory has a classical $A d S_{5} \times S^{5}$ solution and argue that it is stable at the string-theory level for small enough radius. The dual 4-d conformal field theory is the infrared limit of the theory on $N$ electric D3-branes coincident with $N$ magnetic D3-branes. We explicitly construct this $S U(N) \times S U(N)$ gauge theory with global $S O(6)$ symmetry and verify that the one-loop term in the beta function vanishes exactly, while the two-loop term vanishes in the large $N$ limit. We find that this theory is related by a certain projection to the maximally supersymmetric Yang-Mills theory suggesting its large $N$ conformal invariance to all orders in perturbation theory.
\end{abstract}

January 1999

$\dagger$ Also at Lebedev Physics Institute, Moscow. 


\section{Introduction}

In recent papers [1,2,30 non-supersymmetric large $N$ gauge theories dual to certain backgrounds of the type 0 string theory [4] were studied. This work was inspired by the recently discovered relations between type II strings and superconformal gauge theories on $N$ coincident D3-branes [5,6.7,8,9], and, in particular, by Polyakov's suggestion [10] (building on his earlier work [11]) that the type 0 string theory in dimensions $D \leq 10$ is a natural setting for extending this duality to non-supersymmetric gauge theories.

The type 0 string [ [4 has world sheet supersymmetry but no space-time supersymmetry. In both type $0 \mathrm{~A}$ and type $\mathrm{OB}$ theory the fermions are completely projected out of the spectrum. The massless bosonic fields are as in the corresponding type II theory (A or B), but with the doubled set of Ramond-Ramond (R-R) fields. The spectrum of type 0 theory also contains a tachyon, but, as suggested in [10,1], its presence does not spoil the application to large $N$ gauge theories. In fact, the tachyon background provides a natural mechanism for breaking the conformal invariance and introducing the renormalization group flow [1, 2, 3].

In [1] the $3+1$ dimensional $S U(N)$ theory coupled to 6 adjoint massless scalars was constructed as the low-energy description of $N$ coincident electric D3-branes. The conjectured dual type 0B background thus carries $N$ units of electric 5 -form flux. The set of equations for a background with a 5-form flux follows from the following action:

$$
\begin{gathered}
S_{0}=-\frac{1}{2 \kappa_{10}^{2}} \int d^{10} x \sqrt{-g}\left[R-\frac{1}{2} \partial_{n} \Phi \partial^{n} \Phi\right. \\
\left.-\frac{1}{4}\left(\partial_{n} T \partial^{n} T+m^{2} e^{\frac{1}{2} \Phi} T^{2}\right)-\frac{1}{4 \cdot 5 !} f(T) F_{n_{1} \ldots n_{5}} F^{n_{1} \ldots n_{5}}+\ldots\right],
\end{gathered}
$$

where $g_{m n}$ is the Einstein-frame metric, $m^{2}=-\frac{2}{\alpha^{\prime}}$, and [1]

$$
f(T)=1+T+O\left(T^{2}\right) .
$$

In [1,2.3] it was shown that, if the tachyon-R-R field coupling function $f(T)$ has a minimum, then there exists an asymptotically free type 0 background with the tachyon approximately localized near the minimum. The tachyon background induces a radial variation of the dilaton $\Phi$ which has the physical interpretation of renormalization group flow. The Einstein metric is approximately $A d S_{5} \times S^{5}$, while the effective gauge coupling evolves logarithmically with the scale [2,3]. In [2,3] the infrared behavior of the background was

also considered. It turns out that there is a fixed point at infinite coupling which, just like the fixed point at zero coupling, is characterized by the $A d S_{5} \times S^{5}$ Einstein frame metric 
[3]. We argued that there is a renormalization group trajectory extending all the way from the zero coupling fixed point in the UV to the infinite coupling fixed point in the IR.

In this paper we instead consider a background of type 0 theory which has exact conformal invariance: in fact, a line of fixed points. The existence of such a background was pointed out already in [1]: it is the near-horizon region of the self-dual 3-brane of type 0B theory which, just as in the type IIB case [12], turns out to be $A d S_{5} \times S^{5}$. In the type IIB case the dual gauge theory is $\mathcal{N}=4$ SYM [7] but in the type 0B case it is different, and its investigation is the main purpose of this paper.

The self-dual 3-brane is constructed by stacking equal numbers $N$ of coincident electric and magnetic 3-branes. We will show that the non-supersymmetric theory on this stack is the $S U(N) \times S U(N)$ gauge theory coupled to 6 adjoint scalars of the first $S U(N), 6$ adjoint scalars of the second $S U(N)$, and fermions in the bifundamental representations 4 Weyl fermions in the $(\mathbf{N}, \overline{\mathbf{N}})$ and 4 Weyl fermions in the $(\overline{\mathbf{N}}, \mathbf{N})$. We will argue that this theory is conformal in the large $N$ limit. Since it has the maximal global $S O(6)$ invariance, this theory is not of the kind that occurs on the D3-branes of type IIB theory placed at the orbifold singularity of $R^{6} / \Gamma$ [13, 14, 15, 16] (the latter theories are dual to the $A d S_{5} \times S^{5} / \Gamma$ backgrounds of type IIB theory [14,15]).

In section 2 we consider the classical equations for a dyonic type 0B 3-brane and find that for equal electric and magnetic charges there is an $A d S_{5} \times S^{5}$ near-horizon solution. In section 3 we discuss the dual field theory and show that the 1-loop beta function vanishes exactly, while the 2-loop correction vanishes in the large $N$ limit. We further argue that all higher order terms vanish in the large $N$ limit because the field theory can be interpreted as a $Z_{2}$ projection of the $\mathcal{N}=4 U(2 N)$ SYM theory by $(-1)^{F}$ combined with a $Z_{2}$ gauge group twist. In section 4 we discuss the analogy between this projection and a similar $(-1)^{F}$ projection which connects the type II and type 0 string theories compactified on a circle [4, 17, 18, 19].

\section{The $A d S_{5} \times S^{5}$ background of type 0B theory}

Lets us parametrize the 10-d string-frame and Einstein-frame metric as in [1] ( $\mu=$ $0,1,2,3$ are the 4 -d indices)

$$
d s^{2}=e^{\frac{1}{2} \Phi} d s_{E}^{2}, \quad d s_{E}^{2}=e^{\frac{1}{2} \xi-5 \eta} d \rho^{2}+e^{-\frac{1}{2} \xi} d x^{\mu} d x^{\mu}+e^{\frac{1}{2} \xi-\eta} d \Omega_{5}^{2} .
$$

In terms of these variables the effective action describing radial evolution of the fields corresponding to (1.1) becomes [1]

$$
S=\int d \rho\left[\frac{1}{2} \Phi^{\prime 2}+\frac{1}{2} \xi^{\prime 2}-5 \eta^{\prime 2}+\frac{1}{4} T^{2}+U(\Phi, \xi, \eta, T)\right],
$$




$$
U=-\frac{1}{2} T^{2} e^{\frac{1}{2} \Phi+\frac{1}{2} \xi-5 \eta}-20 e^{-4 \eta}+h(T) e^{-2 \xi} .
$$

Here $\alpha^{\prime}=1$, and $\Phi, \xi, \eta$ and $T$ are functions of $\rho$. For the case of the electric 5-form background, it was shown in [1] that $h(T)=Q^{2} f^{-1}(T)$, where $f(T)$ is the tachyon-5form coupling function (1.2) and $Q$ is the electric charge. Generalizing to the presence of magnetic 5-form charge $P$ is straightforward, and we find that

$$
h(T)=P^{2} f(T)+Q^{2} f^{-1}(T)
$$

As in the familiar dilaton-vector coupling case, the potential that originates from the $f(T) F_{5} F_{5}$ term in the full $D=10$ action is invariant under the electro-magnetic duality $P \leftrightarrow Q, f \leftrightarrow f^{-1}$.

The function $h(T)$ has an extremum at $T=T_{0}$ such that $f\left(T_{0}\right)=Q / P$ (we assume that $f(T), Q, P$ are non-negative). For $Q=P(\sim N)$ we thus get $f\left(T_{0}\right)=1$ which has a solution $T_{0}=0$ (cf. (1.2)). Then the tachyon equation is solved by $T=0$, the dilaton equation is solved by $\Phi=\Phi_{0}$, and the equations for $\xi, \eta$ are solved by the usual $A d S_{5} \times S^{5}$ background (note that $h(0)=2 Q^{2}$ for $P=Q$ )

$$
\xi=\ln (2 Q)+\ln \rho, \quad \eta=\ln 2+\frac{1}{2} \ln \rho .
$$

This is the near-horizon $\left(2 Q e^{\Phi_{0}} \rho \gg 1\right)$ limit of the full 3-brane metric. After the change of variables $\rho=u^{-4}$, the $A d S_{5} \times S^{5}$ metric is

$$
d s_{E}^{2}=R_{0}^{2}\left(\frac{d u^{2}}{u^{2}}+\frac{u^{2}}{2 R_{0}^{4}} d x^{\mu} d x^{\mu}+d \Omega_{5}^{2}\right), \quad R_{0}^{2}=\frac{1}{2}(2 Q)^{1 / 2} .
$$

The 4 -th power of the radius $R$ of the corresponding string-frame metric, i.e. $\frac{1}{2} e^{\Phi_{0}} Q$, is proportional to the ' $\mathrm{t}$ Hooft coupling $N g_{\mathrm{YM}}^{2}$ of the gauge theory on the stack of $N$ electric D3-branes and $N$ magnetic D3-branes.

An important issue is that of stability of the background (cf. [20,21]). Considering small perturbations of the $A d S_{5} \times S^{5}$ background in the $T$-direction 1 we learn that they are no longer tachyonic for sufficiently small $Q e^{\Phi_{0}}$. Indeed, the leading quadratic terms in the fluctuation of $T$ near $T_{0}=0$ in the potential $U$ become:

$$
U_{2}=\frac{1}{4 \rho^{2}}\left[-\frac{1}{16}\left(2 Q e^{\Phi_{0}}\right)^{1 / 2}+f^{\prime 2}(0)\right] T^{2}+O\left(T^{3}\right) .
$$

1 The perturbations of the scalar $T$ near $T=0$ do not mix with the dilaton-graviton perturbations since the background value of $F_{5}^{2}$ is zero in the self-dual 3-brane case. In general, the mixing of $T$ and the R-R potential $C_{4}$ fluctuations is already effectively accounted for in $U$ (see [22] for a discussion of stability of a similar dilaton-vector system in 4 dimensions). 
Thus, the background is stable if $16 f^{\prime 2}(0) \geq\left(2 Q e^{\Phi_{0}}\right)^{1 / 2}$, which is satisfied for small enough $Q e^{\Phi_{0}} \sim N g_{\mathrm{s}}$ since $f^{\prime}(0)=1$. More precisely, the stability condition for a scalar field with mass $M^{2}$ in $A d S_{5}$ space-time with radius $R$ is [20]: $M^{2} \geq-4 R^{-2}$, i.e. in the present case $M^{2} \geq-8\left(2 Q e^{\Phi_{0}}\right)^{-1 / 2}$. Here the effective mass parameter (measured in the string frame, $\left.\alpha^{\prime}=1\right)$ is $M^{2}=-2+32 f^{\prime 2}(0)\left(2 Q e^{\Phi_{0}}\right)^{-1 / 2}$, so the stability condition is actually

$$
4+16 f^{\prime 2}(0) \geq\left(2 Q e^{\Phi_{0}}\right)^{1 / 2} .
$$

Note that, once one is allowed to consider the $A d S_{5}$ radius to be of order of $\sqrt{\alpha^{\prime}}$, one in principle does not need the $f(T)$ coupling to achieve the stability of the string tachyon in the $A d S_{5} \times S^{5}$ background. However, the coupling function $f(T)$ is non-trivial in type oB theory, and since $f^{\prime}(0)=1$ [1], its contribution to the above condition is considerable. Explicitly, we get $\frac{1}{2} Q e^{\Phi_{0}}=4 \pi g_{\mathrm{s}} N \alpha^{\prime 2}=g_{\mathrm{YM}}^{2} N$, and thus the following stability condition on the 't Hooft coupling: $g_{\mathrm{YM}}^{2} N<100$.

The stability is related to the fact that the $T=0$ solution is an attractor: a generic solution with $T=T^{(0)}$ at $\rho=0$ evolves to the $T=0$ solution at large $\rho$. This is easy to see explicitly, e.g., by approximating $f(T)$ by $e^{T}$ which is equal to $f_{2}(T)=1+T+\frac{1}{2} T^{2}$ at small $T$. Then $h(T)=2 Q^{2} \cosh T$ which indeed gives a positive shift of the coefficient of the $T^{2}$ term in the potential. Let us note in passing that the choice $f(T)=e^{T}$, which is in agreement with the perturbation-theory result $f_{2}(T)$ of [1] at small $T$ may have more general significance. It is a natural definition of the R-R - tachyon coupling consistent with the expected electric-magnetic 5 -form duality of the type $0 \mathrm{~B}$ theory. In fact, consideration of the D-brane action couplings in [1] implies that the sign of the tachyon tadpole should be changed when changing from the electric to the magnetic 3-brane. Thus, if we require that under the 5 -form duality $T \rightarrow-T$, then the $\mathrm{R}$ - $\mathrm{R}$ - tachyon coupling is fixed to be $e^{T} F_{5} F_{5}$. It is not completely clear, however, whether the massless field/tachyon effective action has to be symmetric under the duality.

A stability criterion similar to (2.8) was already found in [1] where it was noted that it is opposite to the condition of validity of the gravity (leading order in $\alpha^{\prime}$ ) approximation. Indeed, based on (2.8) one may hope that the theory is stable for $Q e^{\Phi_{0}}<O(1)$, but here the curvature of the background is of order 1 in string units and it is not clear a priori whether the effective gravity approximation can be trusted in the first place. We would like to argue, though, that it indeed can be trusted due to the special nature of the $\operatorname{Ad} S_{5} \times S^{5}$ solution.

As we explained in [1], the tree-level effective action of the type $0 \mathrm{~B}$ theory coincides with that of the type IIB theory on the subsector of bosonic fields of the type IIB theory. Since the present self-dual solution has $T=0$ and $F_{5}=\tilde{F}_{5}$ (i.e. the non-vanishing fields 
belong to the spectrum of type IIB theory), we are in a position to argue that our solution is exact to all orders in $\alpha^{\prime}$ just as it is in type IIB theory [23]. The stability condition is also expected to hold (at least qualitatively) since the higher-order in $\alpha^{\prime}$ corrections to the $T^{2}$ term in the effective action are likely to involve powers of the Weyl tensor, $F_{5}^{2}$ and the Ricci scalar which all vanish in the $A d S_{5} \times S^{5}$ background. If one accepts this argument, then one is led to the conclusion that the dual field theory which lives on the stack of D3-branes is a stable large $N$ CFT as long as the 't Hooft coupling $N g_{\mathrm{YM}}^{2}$ is not very large: less than a number of order $10^{2}$. In the next section we present a weak-coupling analysis that provides some support for this conclusion.

We conclude that while the flat space vacuum is unstable in type 0 string theory, the $A d S_{5} \times S^{5}$ with self-dual 5 -form flux should be a stable background for sufficiently small radius. The $\mathrm{R}-\mathrm{R}$ charge works to stabilize the tachyon as proposed in [1]. Our conclusions are independent of the details of the tachyon - R-R coupling function $f(T)$ : any function such that $f(0)=1$ and $f^{\prime}(0)=1$ leads to the stable $A d S_{5} \times S^{5}$ background. These properties of $f(T)$ are obviously satisfied by (1.2), and therefore our conclusions appear to be robust.

\section{Analysis of the Field Theory}

The GSO projections for open strings connecting the D-branes of type 0 theory were considered in 24, 四 2 In considering parallel like-charged D-branes we find the same bosonic spectrum as in the corresponding type II theory, but the fermions are excluded. Thus, for $N$ parallel electric D3-branes we get the $U(N)$ gauge theory coupled to 6 adjoint scalars [1]. Exactly the same theory is found in the case of $N$ parallel magnetic D3-branes. Now we would like to stack $N$ parallel electric and $N$ parallel magnetic D3-branes. The new and somewhat surprising feature is that an open string connecting an electric and a magnetic D3-brane is actually a fermion [24]. It is clear that these fermions will transform in the bifundamental representations of the $U(N) \times U(N)$. It is remarkable that, although type 0 theory has no fermions in the bulk, they are present on the dyonic D3-brane.

It follows already from the perturbative analysis that a composite 'self-dual' type 0B D3-brane consisting of an electric D3-brane coincident with a magnetic D3-brane has a number of special properties. From the results of [1] one finds that the tachyon tadpole has an opposite sign for the magnetic D3-brane compared to the electric one; therefore

2 Open string descendants of type 0B theory were constructed by orientifold projection in [25]. 
the tadpole cancels on the self-dual D3-brane. 3 Also, if one uses the annulus calculations to find the net potential between two such parallel branes by adding up the potentials between constituents, one finds that it vanishes exactly by the abstruse identity. Thus, the self-dual D3-branes behave very much like BPS states even though the type 0 theory has no supersymmetry.

The fermionic nature of the strings connecting an electric and a magnetic D3-brane is crucial for this cancellation. The normalization of the fermions in the annulus diagram connecting an electric and a magnetic D3-brane is the same as between two self-dual D3-branes of type IIB theory. In that case the effective field theory is the $\mathcal{N}=4$ supersymmetric $U(2)$ broken to $U(1) \times U(1)$. Each adjoint of $U(2)$ produces fields with charges $(1,-1)$ and $(-1,1)$ under the $U(1) \times U(1)$. Since there are 4 adjoint Weyl fermions in the $\mathcal{N}=4$ supermultiplet, we find 4 bifundamental fermions of each type. Generalization to $N>1$ is straightforward: the field theory on $N$ electric and $N$ magnetic D3-branes of type 0B theory contains 4 Weyl fermions in the $(\mathbf{N}, \overline{\mathbf{N}})$ of $U(N) \times U(N)$ and 4 Weyl fermions in the $(\overline{\mathbf{N}}, \mathbf{N})$.

The theory with the field content derived above is quite special. First, it has equal numbers of bosonic and fermionic degrees of freedom, $16 N^{2}$, and thus no induced one-loop cosmological constant or quartic divergence. One also finds that the two coefficients in the (one-loop) gravitational trace anomaly are equal, i.e. there is a single central charge as in the $\mathcal{N}=4 \mathrm{SYM}$ theory. As we explain below, the theory is actually a supersymmetry breaking projection of the $\mathcal{N}=4$ SYM theory.

We assume as usual that the $U(1)$ factors decouple in the infrared and consider the $S U(N) \times S U(N)$ theory with the field content derived above. 1 Based on our conjecture

3 Indeed, the resulting self-dual D3-brane action is then decoupled from the tachyon and from the second R-R potential $\bar{C}_{4}$ with anti-selfdual $F_{5}$ [1], and so is essentially the same as in type IIB. In general, the BI part of the action is $\int d^{4} x\left(1+\frac{1}{4} q \bar{q} T+\ldots\right) e^{-\Phi} \sqrt{-\operatorname{det}\left(G_{\alpha \beta}+\partial_{\alpha} X \cdot \partial_{\beta} X+2 \pi F_{\alpha \beta}\right)}$, where $q=\bar{q}=1$ for the electric, $q=-\bar{q}=1$ for the magnetic and $q=2, \bar{q}=0$ for the self-dual D3-brane. The coefficient of the tachyon tadpole was found in [四], and then on reparametrization invariance (and $T$-duality) grounds one expects that the tachyon function $k(T)=1+\frac{1}{4} q \bar{q} T+O\left(T^{2}\right)$ multiplies the $\sqrt{-\operatorname{det}(G+F)}$ in the BI action. We have checked this by directly computing the coefficient of the $T F_{\alpha \beta} F^{\alpha \beta}$ term from the corresponding disk diagram. This calculation was independently carried out in the recent paper [26] where it was also conjectured and then verified that the tachyon coupling function must appear in front of the square root in BI action; in [26] the coefficient of the $T^{2}$ term in $k(T)$ was found to be $\frac{3}{32}$ in the electric $(q=\bar{q}=1)$ case.

4 Requiring the bosons to belong to the adjoint representation of $S U(N) \times S U(N)$ while keeping fermions in the bifundamental representations now implies that the number of bosonic and fermionic degrees of freedom match only approximately, in the large $N$ limit. 
that this field theory is dual to the $A d S_{5} \times S^{5}$ background of type 0B theory, we expect that it is conformal. Indeed, it is not hard to show that the one-loop beta function cancels. The one-loop coefficient $b_{1}$ in a gauge theory with group $G$ coupled to scalars transforming in a representation $S$ and (2-component) Weyl fermions transforming in a representation $F$ is

$$
b_{1}=\frac{11}{3} C_{2}(G)-\frac{1}{6} T_{2}(S)-\frac{2}{3} T_{2}(F)
$$

where $T_{2}$ is the Dynkin index of the representation, $\operatorname{Tr}\left(T_{A} T_{B}\right)=T_{2} \delta_{A B}$. For the adjoint representation of $S U(N) T_{2}=C_{2}(G)=N$, while for the fundamental representation $T_{2}=$ 1/2. As far as each $S U(N)$ is concerned, we have 6 adjoint scalars and $8 N$ fundamental Weyl fermions. It follows from (3.1) that the 1-loop beta function vanishes for each of the two gauge couplings. In fact, we will set the two gauge couplings equal.

The two-loop gauge coupling beta function coefficient is [27,28]

$$
\begin{aligned}
b_{2}= & \frac{34}{3}\left[C_{2}(G)\right]^{2}-\sum\left[2 C_{2}(S)+\frac{1}{3} C_{2}(G)\right] T_{2}(S) \\
& -2 \sum\left[C_{2}(F)+\frac{5}{3} C_{2}(G)\right] T_{2}(F)+Y_{4} .
\end{aligned}
$$

Here $C_{2}(R)$ is the eigenvalue of the quadratic Casimir operator $\hat{C}_{2}=T_{A} T_{A}$ in a representation $R$, i.e. $d(R) C_{2}(R)=d(G) T_{2}(R)$, where $d(R)$ and $d(G)$ are the dimensions of the representation and the group. For the fundamental representation of $S U(N) C_{2}(F)=\frac{N^{2}-1}{2 N}$. $Y_{4}$ is the contribution of the Yukawa couplings [28],

$$
Y_{4}=\frac{1}{d(G)} \operatorname{Tr}\left[\hat{C}_{2}(F) Y_{I} Y_{I}^{\dagger}\right]
$$

where $Y_{I}$ is the matrix determining the Yukawa terms in the Lagrangian, $\Psi \zeta Y_{I} \Psi \mathcal{X}^{I}+$ h.c., where $\zeta=i \sigma_{2} ; \Psi$ is the full set of 2-component fermions and $\mathcal{X}$ is the set of scalars. We are assuming that, as in the $\mathcal{N}=4$ SYM theory, the Yukawa couplings are equal to the gauge coupling constant.

The sums in (3.2) are over irreducible representations. Since the gauge group here is not simple and the fermions are not singlets in both of the groups (and thus 'intertwine' the renormalizations of the two gauge couplings), there is an important subtlety. In general, for a product group with couplings $g_{1}$ and $g_{2}$ one should make make the replacement 28]: $g^{4} C_{2}(F) T_{2}(F) \rightarrow \sum_{k, l=1,2} g_{k}^{2} g_{l}^{2} C_{2}^{(k)}(F) T_{2}^{(l)}(F)$. Since we set $g_{1}=g_{2}=g$, the contribution of the term $-2 \sum C_{2}(F) T_{2}(F)$ in (3.2) is thus effectively doubled, i.e. it is $2 \times 2 \frac{N^{2}-1}{2 N} \times \frac{1}{2} \times 8 N$. As a result,

$$
b_{2}=8-24 N^{2}+Y_{4} \text {. }
$$


This may be compared with the 2-loop coefficient in the $\mathcal{N}=4$ SYM theory [27,29], where the Yukawa contribution is important for the cancellation, $b_{2}=-24 N^{2}+Y_{4}=0$.

To compute $Y_{4}$ in our case let us specify the structure of the interaction terms in the action more explicitly. We shall assume that, like the $\mathcal{N}=4$ SYM theory, our theory has a global $S U(4)$ symmetry. The fermions $\Psi=\left(\psi, \psi^{\prime}\right)$ in the representations $(\overline{\mathbf{N}}, \mathbf{N})$ and $(\mathbf{N}, \overline{\mathbf{N}}$ ) each transform as the $\mathbf{4}$ of $S U(4)$ (and their hermitian conjugates as the $\overline{\mathbf{4}}$ ), while the adjoint scalars $\mathcal{X}=\left(X, X^{\prime}\right)$ each transform as the $\mathbf{6}$ of $S U(4)$. Then the couplings of the fermions to the $S U(N) \times S U(N)$ gauge field $\left(A, A^{\prime}\right)$ are, symbolically, $A\left(\psi^{\dagger} \psi+\psi^{\prime} \psi^{\prime \dagger}\right)$ and $A^{\prime}\left(\psi^{\prime \dagger} \psi^{\prime}+\psi \psi^{\dagger}\right)$, while the Yukawa couplings are $X_{a b} \psi^{a} \zeta \psi^{\prime b}+h . c$. and $X_{a b}^{\prime} \psi^{a} \zeta \psi^{\prime b}+h . c$., where $a, b=1,2,3,4$. The value of the Yukawa contribution $Y_{4}$ then turns out to bel

$$
Y_{4}=24 \times 2 \times 2 N \times \frac{d(F)}{d(G)}\left[C_{2}(F)\right]^{2}=96 C_{2}(F) T_{2}(F)=24\left(N^{2}-1\right) .
$$

As a result, the leading $O\left(N^{2}\right)$ term in (3.4) cancels out and we have $b_{2}=-16.6$ Thus, the theory is 2-loop finite in the large $N$ limit.

In fact, we believe that the non-supersymmetric $U(N) \times U(N)$ theory we are considering can obtained from the $\mathcal{N}=4$ supersymmetric $U(2 N)$ SYM theory by twisting it with $(-1)^{F}$ in a very similar way to how the orbifold $Z_{2}$ theory is obtained [13. The arguments of [16] may then explain why this theory is conformal in the planar limit.

In the orbifold case of [13] the $Z_{2}$ reverses the sign of four of the scalar fields $X$ and also of their fermionic partners. If we think of the $\mathcal{N}=4$ multiplet as an $\mathcal{N}=2$ vector multiplet plus a hypermultiplet, then the $Z_{2}$ reverses the sign of the hypermultiplet fields. Now, break up the $2 N \times 2 N$ matrices into four $N \times N$ blocks. On the diagonal blocks we keep the fields invariant under the $Z_{2}$, i.e. the vector multiplets. On the off-diagonal blocks we keep the non-invariant fields, i.e. the hypermultiplets. This produces the $\mathcal{N}=2$ supersymmetric $U(N) \times U(N)$ theory coupled to two bifundamental hypermultiplets [13]. In effect, we are keeping the fields invariant under the change of sign of the hypermultiplet accompanied by the $U(2 N)$ gauge transformation $\mathcal{I}$ which is the conjugation by $\left(\begin{array}{cc}I & 0 \\ 0 & -I\end{array}\right)$, where $I$ is the $N \times N$ identity matrix.

5 The $S U(4)$ part of the Yukawa matrix $Y$ is the same as in the $\mathcal{N}=4 \mathrm{SYM}, \delta_{c}^{a} \delta_{d}^{b}-\delta_{d}^{a} \delta_{c}^{b}$, and the trace of its square is again 24. Note that all the Yukawa couplings (involving both sets of the scalar fields) contribute to the renormalization of each of the gauge coupling. Another factor of 2 is related to the 'off-diagonal' structure of the Yukawa matrix.

6 This is similar to what happens in the 'orbifold' field theories of [14, 15, 16, as discussed explicitly at the 2-loop level in [30]. 
In our case we take the $Z_{2}$ action to be $\mathcal{I} \cdot(-1)^{F}$, i.e. the $U(2 N)$ gauge transformation is accompanied by the change of sign of all the fermionic fields. Now on the diagonal blocks we keep the invariant fields: the gauge field and the 6 scalars. On the off-diagonal blocks we keep the 4 Weyl fermions. This gives the $U(N) \times U(N)$ theory with 6 scalar adjoints of each $U(N)$, and 8 bifundamental Weyl fermions. The meaning of the $\mathcal{I}$ transformation $\left(\begin{array}{cc}A & B \\ C & D\end{array}\right) \rightarrow\left(\begin{array}{cc}A & -B \\ -C & D\end{array}\right)$ is that it projects out the states corresponding to open strings connecting $N$ electric and $N$ magnetic branes; combining it with $(-1)^{F}$ then allows back the fermionic connecting strings.

\section{Discussion}

One fascinating application of the AdS/CFT correspondence is towards the classification of 4-dimensional conformal gauge theories. For example, interesting large $N$ CFT's with less than maximal supersymmetry are found in the type IIB setting when the $S^{5}$ is replaced by orbifolds of $S^{5}$ [14, 15] or by other Einstein manifolds [31]. Certain non-supersymmetric large $N$ CFT's whose field theoretic formulation is less transparent were studied in [32]. In this paper we propose another route towards constructing nonsupersymmetric large $N$ CFT's: by looking for dual backgrounds of the type 0B theory. The simplest, and most symmetric, background is $A d S_{5} \times S^{5}$. Here we have found the dual gauge theory which implements the corresponding $S O(2,4) \times S O(6)$ symmetry. Just like most non-supersymmetric orbifold CFT's [14,15,30], this theory appears to be conformal only in the large $N$ 't Hooft limit. If $N$ is kept finite then the 2-loop beta function does not cancel exactly.

We have shown that our CFT may be obtained by a $\mathcal{I} \cdot(-1)^{F}$ projection of the $\mathcal{N}=4$ SYM theory. The $(-1)^{F}$ projection is related to the general fact that the type 0 theory is a $(-1)^{F}$ orbifold ( $F$ is the full space-time fermion number) of the type II theory [ [4, 17, 18, 19]. Indeed, the space-time fermions, which are the non-invariant states, are projected out. Thus, it is not too surprising that the self-dual 3-brane of type $0 \mathrm{~B}$ theory is related by a similar projection to the self-dual 3-brane of type IIB theory. As we have seen, the full $Z_{2}$ action on the stack of D3-branes includes also the gauge transformation $\mathcal{I}$.

We have argued that the $A d S_{5} \times S^{5}$ background, which exists only for a non-vanishing $\mathrm{R}-\mathrm{R}$ flux, is stable when its radius is of order the string scale or smaller. This suggests that the type 0 theory is not just a formal twisting of type II theory but, in fact, fits naturally into the AdS/CFT duality. Indeed, there is a general relation between type II and type 0 theories which may have new implications in the presence of a (large) number of D-branes or R-R flux. As was pointed out in [18,19], compactifying type II theory 
on a 9 -circle and applying the $(-1)^{F}$ projection in combination with $(-1)^{2 \pi i R_{9} P_{9}}\left(P_{9}\right.$ is momentum operator) gives a theory which interpolates between the type IIB theory at $R_{9} \rightarrow \infty$ and the type 0B theory at $R_{9} \rightarrow 0$. Equivalently, considering type II theory at finite temperature, i.e. compactifying the euclidean time direction with antiperiodic boundary conditions for space-time fermions, one concludes [17] that the type 0 theory is the high temperature limit of the type II theory. Of course, in flat space one expects a Hagedorn phase transition to take place at the temperature of order the string scale, so that the high-temperature limit is formal. It may be interesting, however, to reexamine the issue of the phase transition for backgrounds with R-R flux.

The relation between the type 0 theory and the thermal type II theory suggests a connection between the type 0 scenario for constructing non-supersymmetric gauge theory and the temperature induced supersymmetry breaking proposed by Witten [33]. However, in [33] the temperature was taken to be much lower than the string scale while in the type 0 setting it is much higher. Nevertheless, there are some similarities between the two approaches which may be pursued further.

\section{Note added:}

Using the general expressions [34,35] for 1-loop renormalization of the scalar potential and the Yukawa matrix (in the form given in eq. (3.45) in [35]) we have checked that the quartic scalar coupling and the Yukawa coupling of the non-supersymmetric $S U(N) \times$ $S U(N)$ gauge theory described above are not renormalized at one loop to the leading order in large $N$. $\mathrm{U}$ In contrast to the 1-loop gauge coupling beta-function (but similar to the 2loop one), the 1-loop scalar and Yukawa beta-functions contain non-vanishing subleading terms. We expect a similar pattern of 1-loop renormalizations in other large $N$ CFT's [14,15 obtained by 'orbifolding' the $\mathcal{N}=4$ SYM theory.

As in the $\mathcal{N}=4$ SYM theory (but in contrast to the 'electric' theory [1] which has the same bosonic spectrum but no fermions) here the fermionic contribution cancels not only the $\operatorname{tr}\left([X, X]^{2}\right)$ term but also the (absent in the classical action) term $\operatorname{tr}\left(X^{2} X^{2}\right)$ in the divergent part of the 1-loop effective action. However, in contrast to the $\mathcal{N}=4 \mathrm{SYM}$

7 We are grateful to E. Witten for raising the question about the scalar and Yukawa coupling renormalizations in this theory. 
here these cancellations happen only to leading order in the large $N$ expansion. 8 A similar conclusion is reached for the Yukawa coupling renormalization.

The reason for the breaking of conformal invariance at subleading orders in $1 / N$ can be understood from the string-theory point of view. 0 Type 0 string theory has a nonvanishing partition function on the torus [4], implying the presence of the string-theory 1-loop cosmological constant in the effective action. The tree-level Einstein frame action $S_{0}$ (1.1) should be supplemented by the 1-loop one $S_{1}=c_{1} \int d^{10} x \sqrt{-g} e^{\frac{5}{2} \Phi} w(T)$, where $w(T)=1+a_{1} T+\ldots$ is a function of the tachyon field. In contrast to what happens in the type IIB theory, where $c_{1}=0$ because of the underlying supersymmetry, here the field equations that follow from $S=S_{0}+S_{1}$ no longer have $\Phi=$ const, $T=0$ and thus $A d S_{5} \times S^{5}$ is no longer an exact solution. Thus, as in the electric theory case [3], one should find a non-trivial RG flow in the corresponding world-volume theory. In the present self-dual case the flow is thus suppressed by $1 / N^{2}$.

Note added in proof:

After this paper was completed, Nekrasov and Shatashvili pointed out [36] that the $Z_{2}$ operation $(-1)^{F}$ which changes the sign of all fermion fields is simply the -1 from the center of the $S U(4)$ R-symmetry group. Thus our theory may be viewed as another orbifold of the $\mathcal{N}=4$ SYM theory by a discrete subgroup of the R-symmetry. In view of the results in [16], this strengthens the argument that this theory is exactly conformal in the planar limit. A special feature of this particular orbifold field theory is that it cannot be realized on D3-branes of type IIB theory placed at an orbifold singularity of $R^{6} / \Gamma$.

\section{Acknowledgements}

We are grateful to E. Kiritsis, I. Kogan, H. Liu, V. Periwal, E. Silverstein and E. Witten for useful discussions and comments. The work of I.R.K. was supported in part by the NSF grant PHY-9802484 and by the James S. McDonnell Foundation Grant No. 91-48. The work of A.A.T. was supported in part by PPARC, the European Commission TMR programme grant ERBFMRX-CT96-0045 and the INTAS grant No.96-538.

8 The reasons for the large $N$ cancellation are the same as in the 1-loop gauge coupling betafunction: (i) the traces in the adjoint and fundamental representations $\mathrm{Tr}$ and $\mathrm{tr}$ are related, for large $N$, simply by the factor of $2 N$, and (ii) while in the $\mathcal{N}=4$ SYM one has 4 adjoint fermions, here we have $4 \times 2 N$ types of fermions in the fundamental representation of $S U(N)$. Since in general for a matrix $X$ from the $S U(N)$ algebra $\operatorname{Tr} X^{2}=2 N \operatorname{tr} X^{2}$ but $\operatorname{Tr} X^{4}=2 N \operatorname{tr} X^{4}+6 \operatorname{tr} X^{2} \operatorname{tr} X^{2}$, the quantum effects induce certain trace-squared terms in the scalar potential, but the renormalization of single-trace terms cancels in the large $N$ limit.

9 We are grateful to E. Silverstein for pointing this out to us. 


\section{References}

[1] I.R. Klebanov and A.A. Tseytlin, "D-Branes and Dual Gauge Theories in Type 0 Strings," hep-th/9811035.

[2] J. Minahan, "Glueball Mass Spectra and Other Issues for Supergravity Duals of QCD Models," hep-th/9811156.

[3] I.R. Klebanov and A.A. Tseytlin, "Asymptotic Freedom and Infrared Behavior in the Type 0 String Approach to Gauge Theory," hep-th/9812089.

[4] L. Dixon and J. Harvey, "String theories in ten dimensions without space-time supersymmetry", Nucl. Phys. B274 (1986) 93; N. Seiberg and E. Witten, "Spin structures in string theory", Nucl. Phys. B276 (1986) 272; C. Thorn, unpublished.

[5] I.R. Klebanov, "World volume approach to absorption by nondilatonic branes," Nucl. Phys. B496 (1997) 231, hep-th/9702076; S.S. Gubser, I.R. Klebanov, and A.A. Tseytlin, "String theory and classical absorption by three-branes," Nucl. Phys. B499 (1997) 217, hep-th/9703040.

[6] S.S. Gubser and I.R. Klebanov, "Absorption by branes and Schwinger terms in the world volume theory," Phys. Lett. B413 (1997) 41, hep-th/9708005.

[7] J. Maldacena, "The Large N limit of superconformal field theories and supergravity," Adv. Theor. Math. Phys. 2 (1998) 231, hep-th/9711200.

[8] S.S. Gubser, I.R. Klebanov, and A.M. Polyakov, "Gauge theory correlators from noncritical string theory," Phys. Lett. B428 (1998) 105, hep-th/9802109.

[9] E. Witten, "Anti-de Sitter space and holography," Adv. Theor. Math. Phys. 2 (1998) 253 , hep-th/9802150.

[10] A.M. Polyakov, "The Wall of the Cave," hep-th/9809057.

[11] A.M. Polyakov, "String theory and quark confinement," Nucl. Phys. B (Proc. Suppl.) 68 (1998) 1 , hep-th/9711002.

[12] G.W. Gibbons and P.K. Townsend, "Vacuum interpolation in supergravity via super p-branes", Phys. Rev. Lett. 71 (1993) 3754, hep-th/9307049.

[13] M. Douglas and G. Moore, "D-branes, quivers, and ALE instantons," hep-th/9603167.

[14] S. Kachru and E. Silverstein, "4d conformal field theories and strings on orbifolds," Phys. Rev. Lett. 80 (1998) 4855, hep-th/9802183.

[15] A. Lawrence, N. Nekrasov and C. Vafa, "On conformal field theories in four dimensions," Nucl. Phys. B533 (1998) 199, hep-th/9803015.

[16] M. Bershadsky, Z. Kakushadze and C. Vafa, "String expansion as large N expansion of gauge theories", Nucl. Phys. B523 (1998) 59, hep-th/9803076; M. Bershadsky and A. Johansen, "Large N limit of orbifold field theories," Nucl. Phys. B536 (1998) 141, hep-th/9803249.

[17] J.J. Atick and E. Witten, "The Hagedorn transition and the number of degrees of freedom of string theory", Nucl. Phys. B310 (1988) 291. 
[18] M. Dine, P. Huet and N. Seiberg, "Large and small radius in string theory", Nucl. Phys. B322 (1989) 301; R. Rohm, "Spontaneous supersymmetry breaking in supersymmetric field theories", Nucl. Phys. B237 (1984) 553.

[19] J. Blum and K. Dienes, "Strong / Weak Coupling Duality Relations for Non Supersymmetric String Theories", Nucl. Phys. B516 (1998) 83, hep-th/9707160.

[20] P. Breitenlohner and D.Z. Freedman, "Stability in gauged extended supergravity", Ann. Phys. 144 (1982) 249.

[21] M. Berkooz and S.-J. Rey, "Nonsupersymmetric stable vacua of M theory," hepth/9807200.

[22] M. Cvetič and A. A. Tseytlin, "Charged string solutions with dilaton and modulus fields", Nucl. Phys. B416 (1994) 137, hep-th/9307123.

[23] T. Banks and M.B. Green, "Nonperturbative effects in $A d S_{5} \times S^{5}$ string theory and d = 4 susy Yang-Mills", J. High Energy Phys. 9805 (1998) 002, hep-th/9804170 ; R.R. Metsaev, A.A. Tseytlin, "Type IIB superstring action in $A d S_{5} \times S^{5}$ background", Nucl. Phys. B533 (1998) 109, hep-th/9805028; R. Kallosh and A. Rajaraman, "Vacua of M theory and string theory", Phys. Rev. D58 (1998) 125003, hep-th/9805041.

[24] O. Bergman and M. Gaberdiel, "A Non-supersymmetric Open String Theory and S-Duality," Nucl. Phys. B499 (1997) 183, hep-th/9701137.

[25] M. Bianchi and A. Sagnotti, "On the Systematics of Open String Theories", Phys. Lett. B247 (1990) 517; A. Sagnotti, "Some Properties of Open - String Theories", hepth/9509080; "Surprises in Open-String Perturbation Theory", Nucl.Phys.Proc.Suppl. B56 (1997) 332, hep-th/9702093.

[26] M.R. Garousi, "String Scattering from D-branes in Type 0 Theories", hep-th/9901085.

[27] D.R.T. Jones, "Asymptotic behavior of supersymmetric Yang-Mills theories in the two-loop approximation," Nucl. Phys. B87 (1975) 127; "Charge renormalization in a supersymmetric Yang-Mills theory," Phys. Lett. B72 (1977) 199; E. Poggio and H. Pendleton, "Vanishing of charge renormalization and anomalies in a supersymmetric gauge theory," Phys. Lett. B72 (1977) 200.

[28] M.E. Machacek and M.T. Vaughn, "Two-loop renormalization group equations in a general quantum field theory I: Wave function renormalization," Nucl. Phys. B222 (1983) 83; "II. Yukawa couplings", Nucl. Phys. B236 (1983) 221; "III. Scalar quartic couplings", Nucl. Phys. B249 (1985) 70.

[29] A. Parkes and P. West, "Finiteness in rigid supersymmetric theories", Phys. Lett. B138 (1984) 99.

[30] P. Frampton, "ADS/CFT String Duality and Conformal Gauge Theories," hepth/9812117.

[31] I.R. Klebanov and E. Witten, "Superconformal field theory on threebranes at a CalabiYau singularity," hep-th/9807080; S.S. Gubser, "Einstein manifolds and conformal field theories," hep-th/9807164. 
[32] L. Girardello, M. Petrini, M. Porrati, and A. Zaffaroni, "Novel Local CFT and Exact Results on Perturbations of N=4 Super Yang Mills from AdS Dynamics", hepth/9810126; J. Distler and F. Zamora, "Non-Supersymmetric Conformal Field Theories from Stable Anti-de Sitter Spaces", hep-th/9810206.

[33] E. Witten, "Anti-de Sitter space, thermal phase transition, and confinement in gauge theories," Adv. Theor. Math. Phys. 2 (1998) 505, hep-th/9803131.

[34] T.P. Cheng, E. Eichten and L.-F. Li, "Higgs phenomena in asymptotically free gauge theories," Phys. Rev. D9 (1974) 2259.

[35] I. Jack and H. Osborn, "General background field calculations with fermion fields", Nucl. Phys. B249 (1985) 472.

[36] N. Nekrasov and S. Shatashvili, "On non-supersymmetric CFT in four dimension," hep-th/9902110. 